\section{An Unexpected Cause of Tenosynovitis}

\section{To the Editor:}

Legionella species are significant pathogens with most infections being caused by Legionella pneumoniae and presenting as Legionnaire disease or Pontiac fever ${ }^{1}$. Extrapulmonary legionellosis is uncommon and is often thought to arise from hematogenous spread after primary pulmonary infection ${ }^{2}$. To date, the extrapulmonary sites of infections that have been reported in the literature include spleen, kidneys, liver $^{3}$, infective endocarditis ${ }^{4}$, myositis $^{5}$, cellulitis, and cutaneous abscesses ${ }^{5}$. The majority of extrapulmonary Legionella infections have been described in immunocompromised patients.

A 69-year-old female florist presented to hospital with 1 week of worsening pain and swelling of the right hand, having been treated in the community for presumed seronegative arthritis for the preceding 6 months. Her presentation 6 months earlier was with pain and swelling in her right hand and mild, transient symptoms in the left hand. Clinical examination at that time found synovitis in multiple proximal interphalangeal (PIP) and metacarpophalangeal (MCP) joints and palmar tenosynovitis in the right hand. Otherwise, joint examination was normal. The tenosynovitis was confirmed on ultrasound (US). She was treated with ceftriaxone, but failed to improve. Synovial aspirate was not performed, but she had negative blood cultures. She commenced treatment with methotrexate $10 \mathrm{mg} /$ week and hydroxychloroquine $400 \mathrm{mg}$ /day. However, she had an incomplete response. The flexor tendon compartment was injected with corticosteroids 1 month prior to the current hospital admission with temporary improvement in her symptoms. She did not respond to a 4-day course of prednisolone (50 $\mathrm{mg} /$ day) immediately prior to the hospital presentation. There were no associated respiratory or systemic symptoms, preceding infection, or traumatic injury. She was Australian-born with no recent overseas travel. She was well apart from a history of hypertension.

On admission to hospital, her right hand and the palmar aspect of her wrist were diffusely swollen, erythematous, and warm. Her fingers were held in fixed flexion. There was no other joint involvement. She was afebrile and hemodynamically stable. The remainder of her physical examination was unremarkable. She was initially managed with a further 3 days of 25 $\mathrm{mg}$ prednisolone and continuation of her disease-modifying antirheumatic drugs. These were then ceased because the unilateral findings and failure to
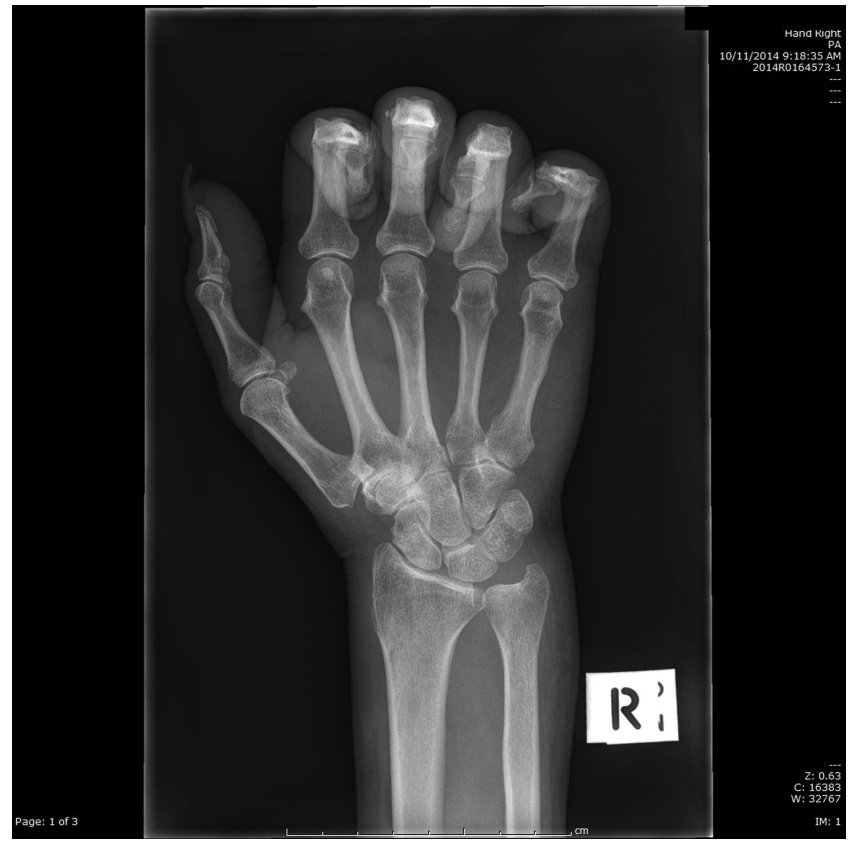

Figure 1. Plain radiograph of right hand demonstrating fixed flexion deformity of the right second, third, fourth, and fifth digits. respond to corticosteroids were felt to be unusual for seronegative arthritis. Her occupation as a florist raised concerns about infection such as sporotrichosis, caused by atypical organisms. Laboratory testing revealed elevated inflammatory markers (erythrocyte sedimentation rate $31 \mathrm{~mm} / \mathrm{h}$, reference range $3-15$, and $\mathrm{C}$-reactive protein $31 \mathrm{mg} / \mathrm{l}$, reference range $0-10$ ). Her full blood count, renal function, and liver function tests were normal. She had 2 sets of negative blood cultures (incubated for 5 days at $35^{\circ} \mathrm{C}$ in soybean casein digest broth) and a negative urine culture. Sputum culture was not performed because she did not have any respiratory symptoms. Rheumatoid factor, anticyclic citrullinated peptide, and antinuclear antibody were negative. Her uric acid was elevated at $0.46 \mathrm{mmol} / 1$ (reference range $<0.35$ ). Radiograph of her right hand showed marked flexion at the PIP joints; however, there were no erosive changes (Figure 1). Radiographs of her left hand, both feet, and chest were normal. US of the right hand and wrist demonstrated flexor tenosynovitis, carpal, and fourth MCP effusions. A synovial fluid aspirate from the right wrist cultured Staphylococcus warneri with a leukocyte count of 0 , which was thought to be a contaminant. There were no crystals.

Magnetic resonance imaging demonstrated extensive subcutaneous edema throughout the right hand with florid tenosynovitis of the flexor tendons and a fluid collection deep to the flexor tendon compartment (Figures 2A and 2B). A washout and synovial biopsy were performed. Synovial tissue histopathology demonstrated active chronic inflammation and no organisms were seen. Initial bacterial (pediatric BACTEC culture), mycobacterial, and fungal cultures of both the synovial tissue and fluid were negative. The synovial tissue was set up on buffered charcoal yeast extract agar and typical Legionella longbeachae colonies were identified on Day 5 by MALDI-TOF (Matrix-Assisted Laser Desorption Ionization Time of Flight Mass Spectrometry; Bruker Corp.). The L. longbeachae was unlikely to be a contaminant given the clinical scenario and her potential occupational exposure. She was prescribed a 6-week course of $750 \mathrm{mg}$ BID ciprofloxacin. She responded well and has regained full hand function with no recurrence of her joint symptoms 6 months after completing the antibiotic course.

L. longbeachae was initially isolated in Long Beach, California, in 1981 and has subsequently been linked to potting mixes ${ }^{6}$. Poor hand hygiene after gardening and exposure to aerosolized organisms have been linked to infection through inhalation or ingestion ${ }^{7}$. To our knowledge, ours is the first reported case of $L$. longbeachae tenosynovitis without preceding injury or clinical pulmonary infection. There has been 1 published case report of septic arthritis in an immunosuppressed patient with rheumatoid arthritis ${ }^{8}$. There have been other reports of soft tissue and musculoskeletal infections with $L$. longbeachae in the context of concomitant pneumonia or direct inoculation through trauma ${ }^{5,9}$. Rare subtypes, such as L. bozemanae, have also been reported to cause septic arthritis ${ }^{10}$.

Legionella infections are often underdiagnosed, especially in extrapulmonary sites. This case highlights the importance of considering rare infective etiologies in the differential diagnosis of subacute or chronic unilateral tenosynovitis, especially in immunocompromised hosts, those with potential occupational exposures, or those not responding to treatment as expected.

LOUISA CHOU*, MBBS(Hons), Rheumatology Department, Royal Melbourne Hospital, Melbourne, Australia; JESSICA DAY*, MBBS(Hons), Rheumatology Department, Royal Melbourne Hospital, Melbourne, Australia; SABINA CICIRIELLO, MBBS, PhD, Rheumatology Department, Royal Melbourne Hospital, Melbourne, Australia. * These authors contributed equally to this work. Address correspondence to Dr. L. Chou, Department of Rheumatology, Royal Melbourne Hospital, 300 Grattan St., Parkville, Melbourne, Australia 3050. E-mail: louisa.chou@monash.edu

\section{REFERENCES}

1. Diederen BM. Legionella spp. and Legionnaires' disease. J Infect 2008;56:1-12.

2. Muder RR, Yu VL. Infection due to Legionella species other than L. pneumophila. Clin Infect Dis 2002;35:990-8. 

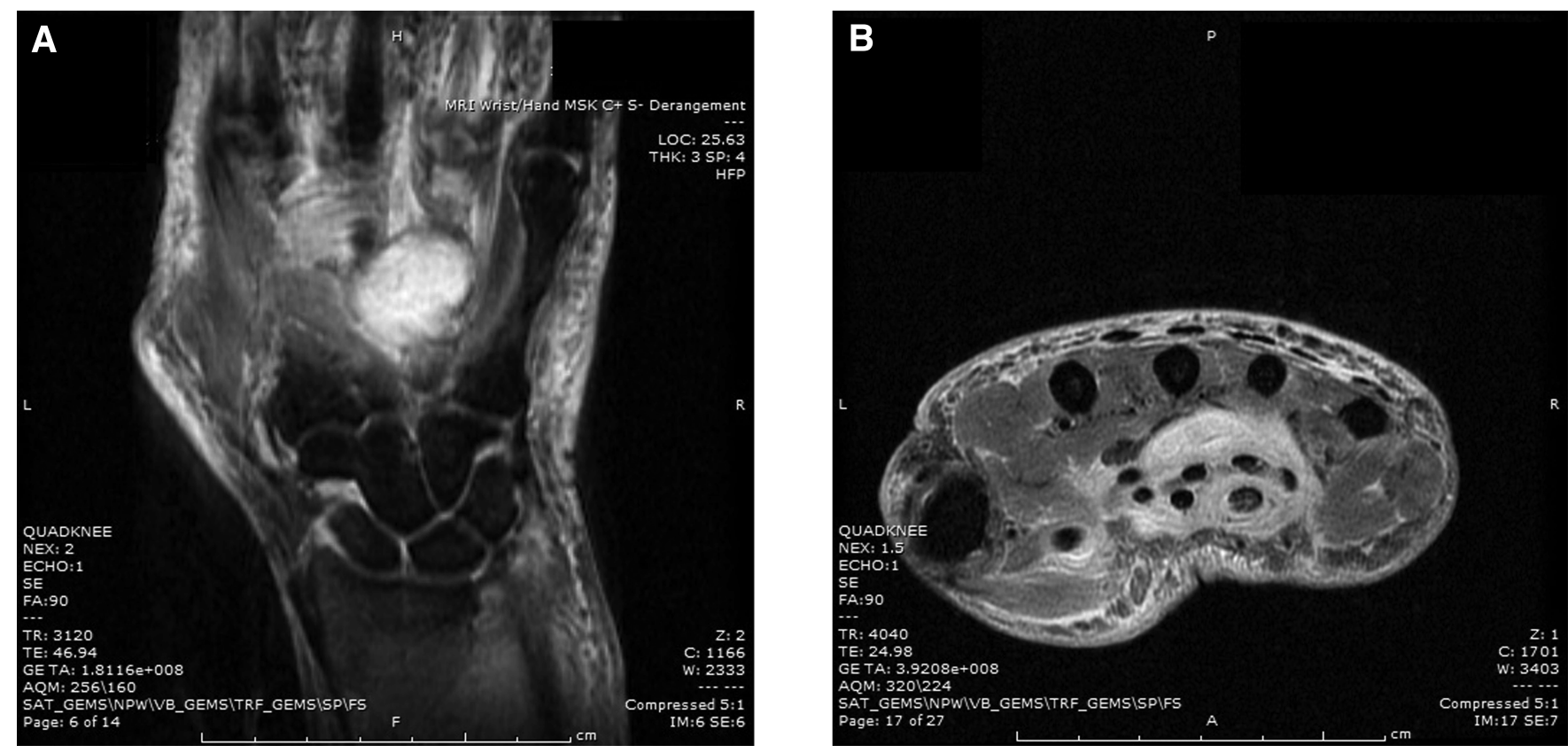

Figure 2. (A and B) Magnetic resonance imaging of the right hand demonstrating extensive subcutaneous edema throughout the hand with florid tenosynovitis of the flexor tendons and a fluid collection deep to the flexor tendon compartment.

3. Monforte R, Marco F, Estruch R, Campo E. Multiple organ involvement by legionella pneumophila in a fatal case of Legionnaires' disease. J Infect Dis 1989;159:809.

4. Leggieri N, Gouriet F, Thuny F, Habib G, Raoult D, Casalta JP. Legionella longbeachae and endocarditis. Emerg Infect Dis 2012;18:95-7.

5. McClelland MR, Vaszar LT, Kagawa FT. Pneumonia and osteomyelitis due to Legionella longbeachae in a woman with systemic lupus erythematosus. Clin Infect Dis 2004;38:e102-e6.

6. Lindsay DS, Brown AW, Brown DJ, Pravinkumar SJ, Anderson E, Edwards GF. Legionella longbeachae serogroup 1 infections linked to potting compost. J Med Microbiol 2012;61:218-22.

7. O'Connor BA, Carman J, Eckert K, Tucker G, Givney R, Cameron $\mathrm{S}$. Does using potting mix make you sick? Results from a
Legionella longbeachae case-control study in South Australia. Epidemiol Infect 2007;135:34-9.

8. Dugar M, Rankin WA, Rowe E, Smith MD. "My foot hurts": a flare of rheumatoid arthritis? Med J Aust 2009;190:392-3.

9. Mentula S, Pentikäinen J, Perola O, Ruotsalainen E. Legionella longbeachae infection in a persistent hand-wound after a gardening accident. J Med Microbiol Case Rep 2014;1-3.

10. Just SA, Knudsen JB, Uldum SA, Holt HM. Detection of Legionella bozemanae, a new cause of septic arthritis, by pcr followed by specific culture. J Clin Microbiol 2012;50:4180-2.

J Rheumatol 2016;43:2; doi:10.3899/jrheum.150968 